\title{
Método de ensayo normalizado del comportamiento de los tejados a la acción combinada de la lluvia y del viento
}

\author{
Standard test method to determine the performance \\ of tiled roofs to wind-driven rain
}

\author{
F. Marín Andrés(*) y Ma. I. Sánchez de Rojas Gómez ${ }^{(*)}$
}

Recepción/Received: 26-III-07

Aceptación/Accepted: 18-VI-07

Publicación online/Online publishing: 30-V-08

\section{RESUMEN}

Para el diseño de los tejados es importante determinar el punto hasta el cual éstos pueden resistirse a la penetración de agua causada por la combinación de viento y lluvia. Un nuevo proyecto de Norma Europeo prEN 15601 (1) especifica un método de ensayo para determinar el comportamiento del tejado frente a la combinación de viento y lluvia.

La acción combinada de viento y lluvia varía considerablemente con la situación geográfica de un edificio y las diferencias asociadas al clima de la lluvia y del viento. El proyecto de norma especifica las condiciones de viento y lluvia y una condición de diluvio para cada una de las tres zonas de Europa: Europa del Norte y Costera, Europa Central y Europa del Sur, cada una subdividida en cuatro condiciones de velocidades de viento y caudal de lluvia para ser aplicadas en los ensayos. El proyecto no contiene la información sobre condiciones aceptables.

Palabras clave: comportamiento de tejados, efecto combinado de lluvia y viento, estanquidad de tejados, túneles de viento.

\section{SUMMARY}

The extent to which roof coverings can resist water penetration from the combination of wind and rain, commonly referred to as wind driven rain, is important for the design of roofs. A new project of European Standard prEN 15601 (1) specifies a method of test to determine the performance of the roof covering against wind driven rain.

The combined action of wind and rain varies considerably with geographical location of a building and the associated differences in the rain and wind climate. Three windrain conditions and one deluge condition covering Northern Europe Coastal, Central Europe and Southern Europe are specified in the project standard, each subdivided into four wind-speeds and rainfall rates to be applied to the test. The project does not contain information on the level of acceptable performance.

Keywords: roof tightness, tiled roof performance, winddriven rain, wind tunnels.

\footnotetext{
(*) Uralita Tejados, S.A. (Madrid, España).

(**) Instituto de Ciencias de la Construcción Eduardo Torroja (CSIC), (Madrid, España).
} 


\section{INTRODUCCIÓN}

La necesidad de una norma europea de comportamiento a la acción combinada de viento y lluvia puede explicarse por:

- Actualmente no hay disponibles métodos de ensayo comunes para validar productos nuevos o tradicionales ni el modo de instalarlos (2). Incluso los productos tradicionales para tejados pueden no comportarse bien si se trasladan a otros mercados donde el clima es diferente (3).

- Es preciso identificar y racionalizar las condiciones climáticas extremas de viento y lluvia existentes en Europa (4).

- Varios países solicitaron a CEN la elaboración de un método de ensayo válido y basado en el acuerdo para desarrollar especificaciones de instalación en diferentes climas, evitando barreras al libre comercio y que proporcionen a fabricantes, instaladores y usuarios finales la confianza en el tejado y en sus componentes.

- Los diferentes diseños de los túneles de viento añaden una complicación adicional a la medida del comportamiento higrotérmico de los tejados (5).

El método de ensayo de lluvia y viento desarrollado sólo será preciso utilizarlo en aquellos estados Miembros en los que exista un requisito escrito de carácter reglamentario o normativo. En el resto de los Estados Miembros el ensayo será opcional.

El borrador de norma ha sido desarrollado por el CEN / TC89 / WG10 / TG4. La mayor parte del desarrollo técnico fue delegado en un Grupo de Laboratorios que incluía fabricantes (Lafarge Roofing, Marley Buildings Services), Centros técnicos y de desarrollo (Building Research
Establishment, UK, CERIB (6), CTTB, Francia, TNO, Holanda) y universidades (Universidad Técnica de Berlin). Se consideraron diferentes tipos de túneles de viento y todos los laboratorios participaron en los ensayos comparativos inter-laboratorios. La financiación fue proporcionada por los fabricantes de tejas, organizaciones de instaladores, organismos nacionales de normalización y los propios laboratorios.

El proyecto de norma, completado por el Grupo de Laboratorios y aprobado por el TG4, que fue elevado al CEN TC89 / WG10 con la solicitud de ser enviado a Encuesta CEN, estuvo a disposición del público para comentarios y voto indicativo (diciembre 2006 - abril 2007).

\section{ENSAYO DE LLUVIA Y VIENTO}

\subsection{Principios}

La estanquidad al agua de los tejados depende de los siguientes factores (4):

- Las condiciones de lluvia y viento sobre el tejado. Los agentes atmosféricos se ven afectados por el edificio y el propio tejado (7).

- La pendiente del tejado y su diseño.

- El diseño de los materiales del tejado y sus especificaciones de instalación.

- El gradiente de presiones a través de la cubierta, asociado a los flujos por el espacio bajo tejas (véase figura 1).

Se denomina muestra de ensayo a un conjunto de tejas instaladas en un tejadillo para ensayo de modo similar a

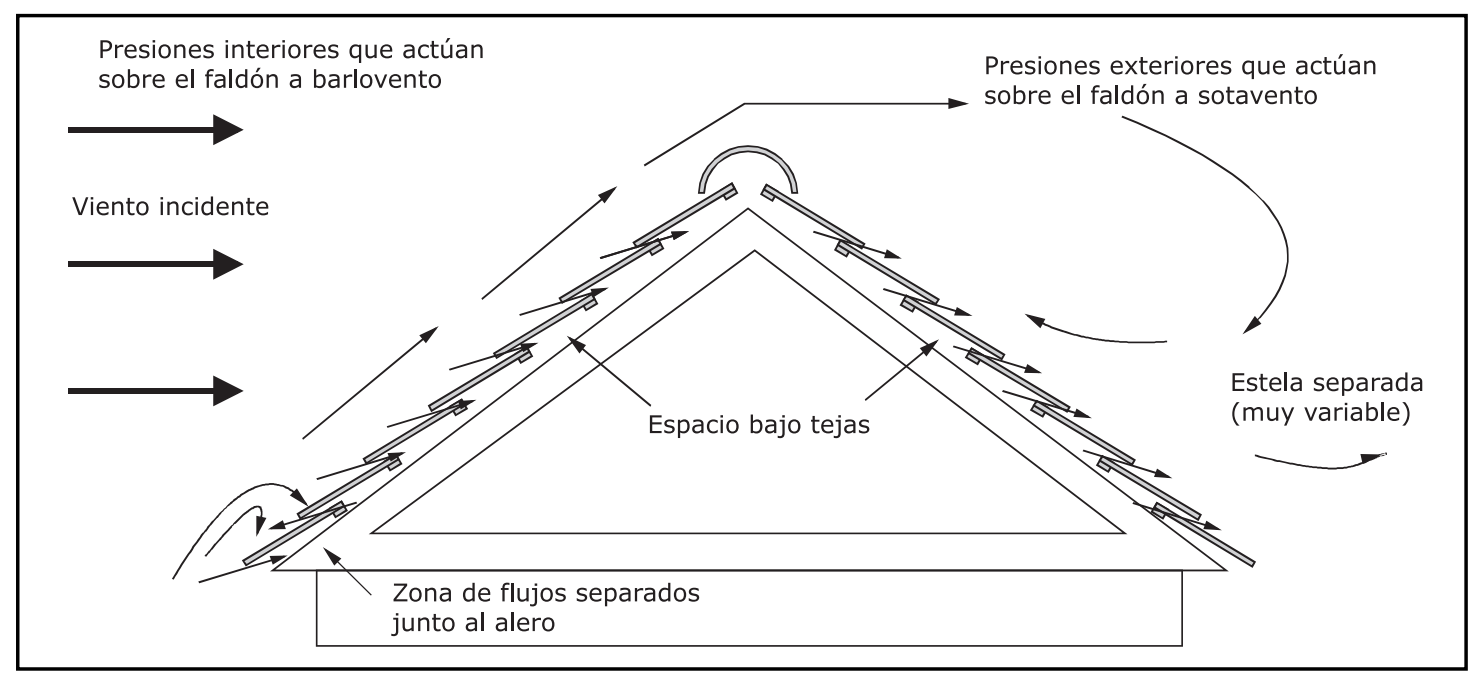

Figura 1. El flujo de viento bajo las tejas determina el gradiente de presiones a través de las tejas 
como se construye un tejado, tomando en consideración pendiente, solape y ensamble. El tejadillo de ensayo se instala en el túnel de viento y se somete a las primeras condiciones de lluvia y viento del ensayo.

En total, se han especificado 3 zonas climáticas europeas (Europa del Norte y costera, Europa Central y Europa del Sur). En cada una hay 4 combinaciones de lluvia y viento designadas de la "A" a la "D". B y D son de ensayo obligatorio, A y $\mathrm{C}$ son opcionales.

Cualquier pendiente del tejado puede ser ensayada y el caudal de agua incidente en la parte superior del conjunto de tejas pude ajustarse para simular cualquier longitud del paño del tejado (por defecto se toman $5 \mathrm{~m}$ adicionales).

Se aplica una succión gradualmente creciente a través del conjunto de tejas en etapas con una duración de 5 minutos cada una. Generalmente, al principio no se produce filtración. A medida que se aumenta la succión se alcanza un punto en que comienza una filtración sustancial. La filtración se observa y se cuantifica. El nivel de succión requerido para que se produzca una filtración significativa a través del conjunto de tejas proporciona una medida del comportamiento de las tejas en las condiciones de lluvia y viento ensayadas (8). La figura 2 muestra un ejemplo de curvas de Filtración frente a la Succión Aplicada para tres productos bajo las mismas condiciones de lluvia y viento. rencia con buen comportamiento reconocido, elegidos por los laboratorios de ensayo o por los organismos nacionales de normalización.

\subsection{Dispositivos de ensayo}

Los dispositivos de ensayo estarán compuestos por:

a) Una cámara de succión sellada al perímetro inferior de la muestra de ensayo y conectada a un aspirador, capaz de crear una diferencia de presión estable mantenida durante un periodo de tiempo de 5 minutos \pm 10 segundos, a través de la muestra de ensayo. La diferencia de presión debe ser medida con una precisión superior al valor mayor de $1 \%$ o 2,5 Pa. Debe disponerse un colector de agua conectado a la cámara de succión para registrar la cantidad de agua filtrada durante cualquier etapa de presión de ensayo.

b) Un sistema de impulsión de aire para crear viento en la superficie exterior de la muestra de ensayo dirigido desde el alero hacia la cumbrera. El viento debe ser horizontal o paralelo a la superficie de la muestra de ensayo inclinada. La velocidad del viento debe medirse con una precisión superior a $0,5 \mathrm{~m} / \mathrm{s}$, la intensidad de las turbulencias del viento incidente debe ser inferior al $10 \%$ en cada punto y la variación espacial de la velocidad del viento no debe superar $\pm 10 \%$ sobre

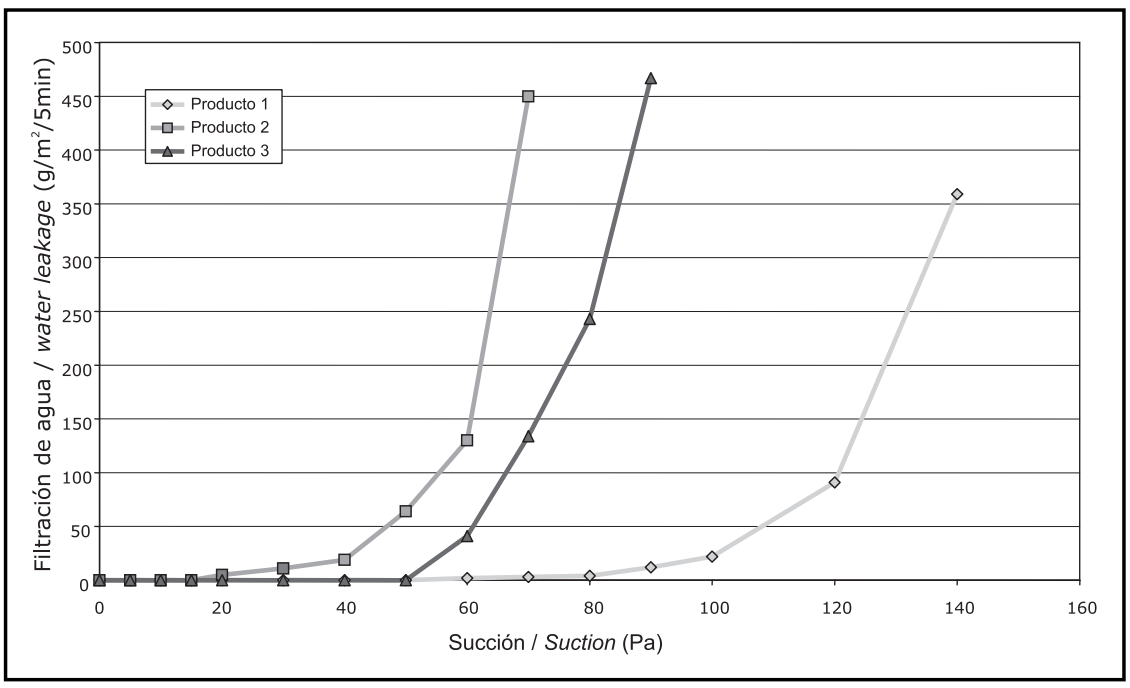

Figura 2. Ejemplo de curvas de Filtración frente a Succión para 3 productos bajo las mismas condiciones de lluvia y viento.

El conjunto de tejas se seca y se prepara para el ensayo de las siguientes condiciones de lluvia y viento.

Los niveles de succión requeridos para producir el inicio de la filtración en cada condición de lluvia y viento son comparados con los resultados de los productos de refe- la muestra de ensayo, tras la calibración del sistema de impulsión.

c) Una instalación capaz de proporcionar un caudal estable de lluvia, tal como establece la tabla 1 para la pendiente del tejado ensayada. La variación espacial 
Tabla 1 / Table 1

Condiciones de viento y lluvia para ensayos

\begin{tabular}{|c|c|c|c|c|c|}
\hline \multicolumn{2}{|r|}{ SUB-ENSAYO / SUB-TEST } & $\mathbf{A}$ & B & C & D \\
\hline \multirow{11}{*}{ 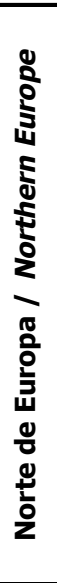 } & $\begin{array}{c}\text { Velocidad del viento / Wind speed } \\
u \mathrm{~m} / \mathrm{s}\end{array}$ & 5 & 13 & 25 & - \\
\hline & Caudal de lluvia / Rainfall $\boldsymbol{R}_{\mathrm{h}} \mathrm{mm} / \mathrm{h}$ & 110 & 60 & 6 & 225 \\
\hline & $\begin{array}{l}\text { Pendiente del tejado / Roof pitch } \\
\alpha^{\circ}\end{array}$ & \multicolumn{4}{|c|}{$\begin{array}{c}\text { Caudal de lluvia sobre la superficie del tejado / Rainfall on } \\
\text { roof surfaces } \boldsymbol{R} \mathrm{mm} / \mathrm{h}\end{array}$} \\
\hline & 15,0 & 124 & 85 & 13 & 217 \\
\hline & 17,5 & 126 & 89 & 14 & 215 \\
\hline & 20,0 & 127 & 92 & 15 & 211 \\
\hline & 25,0 & 129 & 99 & 17 & 204 \\
\hline & 30,0 & 130 & 104 & 19 & 195 \\
\hline & 35,0 & 129 & 109 & 20 & 184 \\
\hline & 40,0 & 128 & 113 & 22 & 172 \\
\hline & 45,0 & 126 & 116 & 23 & 159 \\
\hline \multirow{11}{*}{ 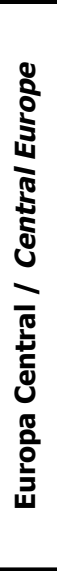 } & $\begin{array}{c}\text { Velocidad del viento / Wind speed } \\
u \mathrm{~m} / \mathrm{s}\end{array}$ & 4 & 10 & 15 & - \\
\hline & Caudal de lluvia / $\boldsymbol{R}$ ainfall $\boldsymbol{R}_{\mathrm{h}} \mathrm{mm} / \mathrm{h}$ & 200 & 130 & 8 & 300 \\
\hline & $\begin{array}{c}\text { Pendiente del tejado / Roof pitch } \\
\alpha^{\circ}\end{array}$ & \multicolumn{4}{|c|}{$\begin{array}{l}\text { Caudal de lluvia sobre la superficie del tejado / Rainfall on } \\
\text { roof surfaces } \boldsymbol{R} \mathrm{t} \mathrm{mm} / \mathrm{h}\end{array}$} \\
\hline & 15,0 & 217 & 167 & 13 & 290 \\
\hline & 17,5 & 219 & 172 & 14 & 286 \\
\hline & 20,0 & 220 & 176 & 15 & 282 \\
\hline & 25,0 & 220 & 185 & 16 & 272 \\
\hline & 30,0 & 219 & 192 & 17 & 260 \\
\hline & 35,0 & 217 & 197 & 18 & 246 \\
\hline & 40,0 & 213 & 202 & 19 & 230 \\
\hline & 45,0 & 207 & 204 & 20 & 212 \\
\hline \multirow{11}{*}{ 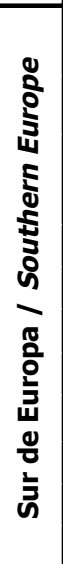 } & $\begin{array}{c}\text { Velocidad del viento / Wind speed } \\
u \mathrm{~m} / \mathrm{s}\end{array}$ & 2 & 8 & 20 & - \\
\hline & Caudal de lluvia / Rainfall $\boldsymbol{R}_{\mathrm{h}} \mathrm{mm} / \mathrm{h}$ & 248 & 166 & 6 & 415 \\
\hline & $\begin{array}{c}\text { Pendiente del tejado / Roof pitch } \\
\alpha^{\circ}\end{array}$ & \multicolumn{4}{|c|}{$\begin{array}{c}\text { Caudal de lluvia sobre la superficie del tejado / Rainfall on } \\
\text { roof surfaces } \boldsymbol{R t} \mathrm{mm} / \mathrm{h}\end{array}$} \\
\hline & 15,0 & 254 & 201 & 11 & 401 \\
\hline & 17,5 & 253 & 206 & 12 & 396 \\
\hline & 20,0 & 252 & 210 & 13 & 390 \\
\hline & 25,0 & 248 & 217 & 14 & 376 \\
\hline & 30,0 & 243 & 222 & 16 & 359 \\
\hline & 35,0 & 235 & 226 & 17 & 340 \\
\hline & 40,0 & 226 & 228 & 18 & 318 \\
\hline & 45,0 & 215 & 229 & 19 & 293 \\
\hline
\end{tabular}

no debe superar $\pm 35 \%$ sobre la superficie de la muestra durante cada etapa de ensayo y el caudal de lluvia no debe variar más del $\pm 2 \%$. El tamaño de las gotitas de lluvia debe ser representativo de la lluvia natural, siendo predominante el intervalo de $0,6 \mathrm{~mm}$ a 2,5 mm de diámetro. Las gotitas de agua introducidas en una corriente de aire de alta velocidad tienden a romperse a lo largo de la distancia, por lo que se recomienda que las gotitas sean introducidas lo bas- tante lejos de la muestra de ensayo para que éste proceso se complete y que las gotitas adquieran la velocidad requerida antes de impactar con la muestra ensayada $(9,10)$. Para calibrar la lluvia que cae directamente sobre la muestra de ensayo se sustituye ésta por un tablero plano que incorpora elementos de medida del agua de lluvia en la superficie superior. Los elementos de medida deben ser, cada uno, de dimensiones entre $0,10 \mathrm{~m}^{2}$ y $0,20 \mathrm{~m}^{2}$ y dispuestos de 
tal manera que no recojan ningún agua de elementos adyacentes durante la calibración. La lluvia debe medirse con una precisión superior al valor mayor entre $3 \%$ y $0,2 \mathrm{~mm} / \mathrm{h}$.

d) Alimentación de agua a la fila superior de la muestra de ensayo, que simula el agua incidente de la zona superior del paño del tejado. La cantidad de agua incidente en la fila superior debe ser medida con una precisión superior al $3 \%$. Deben tomarse precauciones para evitar distribuciones de agua en la zona superior no representativas de los elementos superiores de cobertura que, por ejemplo, puede causar la filtración a través de los ensambles laterales. Como medida preventiva, pueden sellarse los solapes laterales de la fila superior de tejas.

e) Instalación para observar y medir la filtración. La cámara de succión debe estar provista de:

o Una superficie inferior transparente que permita la observación visual con claridad de la naturaleza y posición de las filtraciones que puedan aparecer en el reverso de la muestra durante el ensayo;

o Un dispositivo de recogida y medida del agua filtrada que pueda caer a la cámara de succión desde la muestra durante el ensayo. Para minimizar la ten-

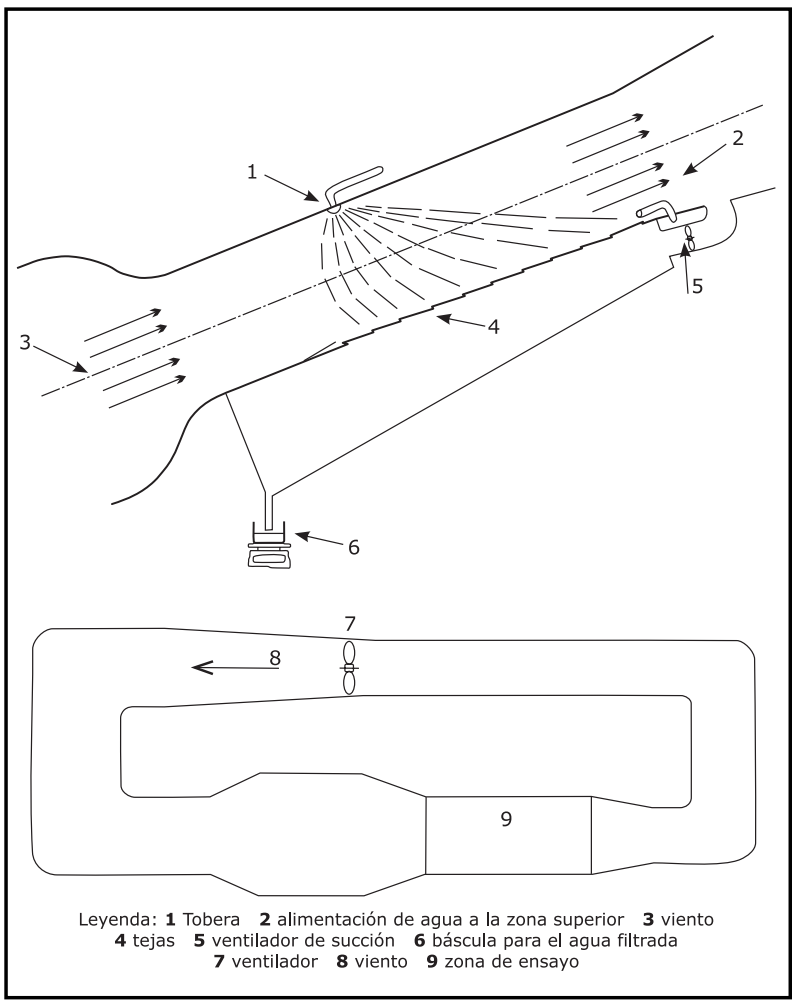

Figura 3. Túnel de viento cerrado con viento paralelo a la muestra de ensayo (planta y sección).

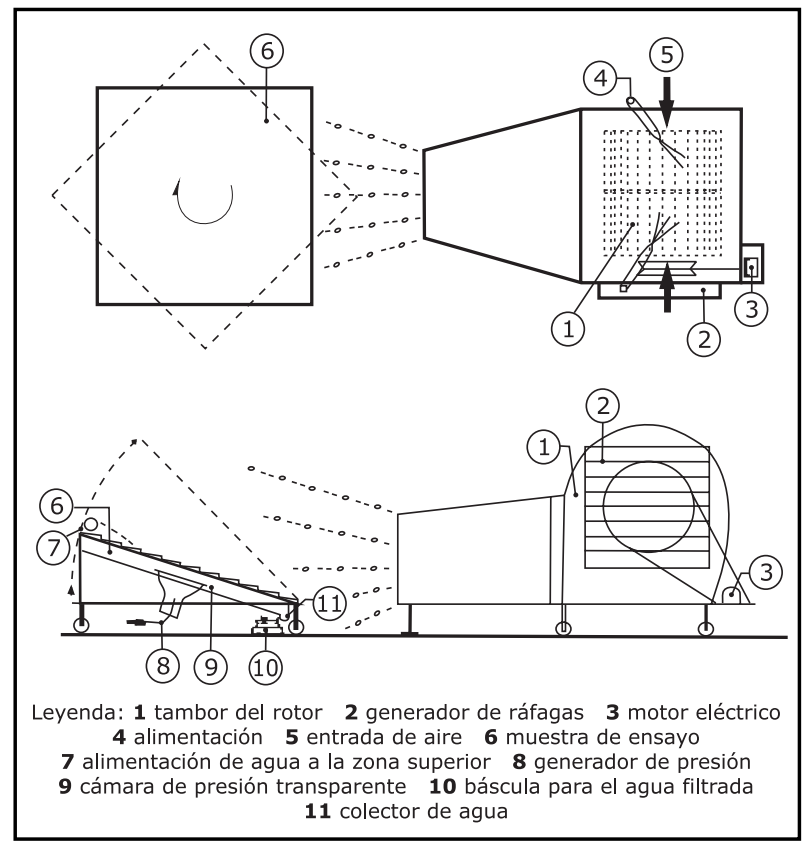

Figura 4. Aparato abierto con viento horizontal.

sión superficial, la absorción y la retención del agua en las superficies internas de la cámara de succión, las superficies serán lisas, no absorbentes e inclinadas en un ángulo vertical de no menos de $15^{\circ}$ de la horizontal hacia el aparato de recogida inferior durante el ensayo.

Ejemplos de dispositivos de ensayo de lluvia y viento se ilustran en la figura 3 (túnel de viento cerrado) y 4 (aparato abierto). En los dispositivos donde la muestra de ensayo se sitúa paralela a la dirección del viento es preciso corregir la velocidad del viento para tener en cuenta el efecto de la pendiente del tejado, multiplicando la velocidad del viento por el factor de de corrección $\mathrm{f}$ (véase tabla 2).

Tabla 2 / Table 2

Factor de corrección para la velocidad del viento según la pendiente del tejado

\begin{tabular}{|c|c|}
\hline $\begin{array}{c}\text { Pendiente del tejado } \\
\text { Roof pitch }\left(^{\circ}\right)\end{array}$ & $\boldsymbol{f}$ \\
\hline 15,0 & 0,85 \\
\hline 17,5 & 0,81 \\
\hline 20,0 & 0,80 \\
\hline 25,0 & 0,76 \\
\hline 30,0 & 0,71 \\
\hline 35,0 & 0,67 \\
\hline 40,0 & 0,60 \\
\hline 45,0 & 0,54 \\
\hline
\end{tabular}




\subsection{Procedimiento de ensayo}

El ensayo debe llevarse a cabo a una temperatura entre 5 ${ }^{\circ} \mathrm{C}$ y $35^{\circ} \mathrm{C}$ con la muestra de ensayo instalada en el dispositivo con la pendiente especificada con los bordes sellados para prevenir el paso de agua o aire hacia o desde la cámara de succión y la superficie seca de las tejas

Se eligen y se aplican de modo continuo las condiciones pertinentes de velocidad del viento, caudal de agua de lluvia y cantidad de agua alimentada en la zona superior, de acuerdo con las especificaciones de ensayo. Un conjunto de ensayos debe consistir en los ensayos $B$ y $D, y$ opcionalmente $\mathrm{A}$ y $\mathrm{C}$, realizados con las combinaciones de lluvia y viento definidas en la figura 5 para las áreas climáticas deseadas:

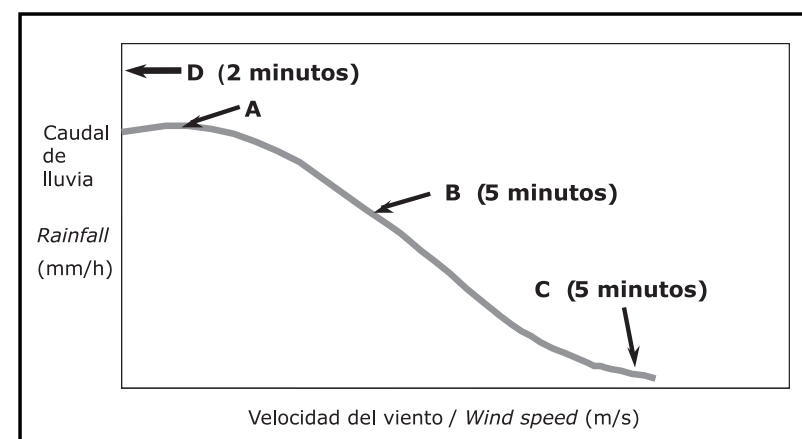

Figura 5 .

- Ensayo A: Velocidad del viento baja, con una lluvia intensa;

- Ensayo B: Velocidad del viento moderada con una lluvia moderada;

- Ensayo C: Velocidad del viento elevada con una lluvia suave;

- Ensayo D: Máxima intensidad de lluvia sin viento (diluvio).

En los ensayos de lluvia y viento combinados ( , B y $C$ ), se mide inicialmente la diferencia de presión con la cámara de succión cerrada y se toma esta diferencia de presión como valor cero de referencia para los siguientes cambios de presión realizados durante el ensayo. A continuación se reduce la presión de la cámara en etapas de, al menos, $5 \mathrm{~Pa}$ y se mantiene cada diferencia de presión durante 5 minutos \pm 10 segundos, hasta que se observan filtraciones. Se mide la cantidad de agua filtrada en cada etapa, o de modo continuo, hasta la velocidad de filtración de referencia.

En el ensayo de diluvio (D) se hace caer el agua de lluvia y el agua alimentada en la zona superior sin viento con la cámara de succión abierta a la atmósfera, durante 2 minutos \pm 10 segundos. Se observa cualquier filtración y se mide la cantidad de agua filtrada.

Agua finamente pulverizada puede pasar por las juntas en ciertos tipos de tejas, produciendo pequeñas cantidades de agua bajo la muestra de ensayo o sobre la superficie de la cámara de succión. Si esto ocurre, debe ser registrado. Esta agua pulverizada puede o no considerarse filtración, dependiendo del criterio adoptado para la filtración.

Se deben tomar medidas para evitar una filtración de agua que suba por debajo de la muestra de ensayo y el drenaje de agua desde la fila inferior del tejadillo, por ejemplo, aplicando una cordón de grasa a lo largo del reverso de todas las tejas por debajo de la zona superior de la fila de tejas o pizarras inferior.

Durante el ensayo, se debe observar continuamente el reverso de la muestra de ensayo para registrar cualquier filtración, la presión, paso de agua pulverizada y humidificación del reverso.

Se deben registrar las cantidades de agua filtradas y su correspondiente diferencia de presión, dentro de cada etapa de ensayo. La descripción de la filtración puede complementarse con diagramas y fotografías.

Se compara el comportamiento del material ensayado con otros materiales de los que se tenga constancia de un comportamiento satisfactorio. Para que el comportamiento sea aceptable, la succión aplicada en el ensayo no debería ser menor que que la aplicada al elemento de referencia con el mismo filtración en idénticas condiciones combinadas de lluvia y viento. Los ensayos comparativos deben realizarse en el mismo laboratorio. La succión aplicada para producir una filtración de $2 \mathrm{~g} /\left(\mathrm{m}^{2} \cdot \mathrm{min}\right)$ durante un ensayo de 10 minutos es la base preferida de comparación del comportamiento.

\subsection{Ensayos comparativos interlaboratorios}

Los ensayos comparativos interlaboratorios han considerado:

- Tejas con una larga historia en una o mas áreas de mercado Europeo:

- Tejas de hormigón perfiladas con ensambles.

- Tejas de hormigón planas con ensambles.

- Tejas de arcilla cocida perfiladas con un solo ensamble.

- Tejas de arcilla cocida planas con doble sistema de ensamble.

- Pizarras de fibrocemento con doble sistema de ensamble. 
- Repetibilidad, los diferentes productos fueron re-instalados y ensayados de nuevo.

- La influencia de la pendiente del tejado, algunos productos se ensayaron a más de una pendiente.

— Una longitud de paño de 6,5 m. por defecto.

Los dispositivos de ensayo con diseños diferentes probablemente producirán resultados de ensayo de lluvia y viento combinados diferentes, pero pueden producir comparaciones consecuentes del comportamiento entre distintos elementos de cubrición de tejados.

En función de los ensayos interlaboratorios, las condiciones de lluvia y viento $B$ y $D$ son obligatorias y las A y $C$ son optativas.

\section{CONCLUSIONES}

El borrador de norma de ensayos del efecto combinado de lluvia y viento, prEN 15601, ha pasado la fase de encuesta CEN, establece:
- Una metodología de ensayo para determinar el comportamiento de los elementos del tejado frente a la acción combinada de la lluvia y del viento.

- Un procedimiento de calibración para conseguir las mismas condiciones de ensayo en cualquier tipo de túnel de viento o aparato abierto $\mathrm{y}$ factores de corrección dependiendo de la pendiente.

- Tres climas europeos diferentes y 4 condiciones severas de lluvia y viento para cada uno de los climas.

- Guía sobre los criterios de ensayo, para facilitar su selección por los Estados Miembro. El ensayo desarrollado solamente será exigido en los Estados Miembros con un requisito por escrito de carácter reglamentario o normativo en el campo de la construcción.

Los ensayos interlaboratorios aseguran la repetibilidad utilizando distintos tipos de dispositivos experimentales, habiendo considerado diversos modelos de tejas con una larga historia de buen comportamiento acreditado en una o más zonas de Europa.

\section{BIBLIOGRAFÍA}

(1) prEN 15601 Hygrothermal performance of buildings. Resistance to wind-driven rain of roof coverings with discontinuously laid small elements. Test method (Nov. 2006).

(2) Blocken, B., Carmeliet, J.: "A review of wind-driven rain research in building science". J. Wind Eng. and Ind. Aerodinamics, vol. 92, 13 (2004) págs. 1079/1130.

(3) Cobos Hernández, F., Marín Andrés, F.: "Problemática en tejados y fachadas de ladrillo visto". Arte y Cemento, 1.889 (2000), pag. $92 / 6$.

(4) Owen, P. R.: Buildings in the wind. Quarterly J. Roy. Met. Soc., 97, 114 (1971), págs. 396/413.

(5) Blocken, B., Carmeliet, J.: "On the accuracy of wind-driven rain measurement in buildings". Building and Env. vol. 14,12 (2006), págs. $1798 / 1810$.

(6) Bresson, J.: "La soufflerie du Cerib: Premiers resultats obtenus sur le comportement des couvertures". J. Wind Eng. and Ind. Aerodinamics. vol. 23 (1986) págs. 283/296.

(7) Lacey, R.E.: Climate and building in Britain, BRE report, (1977).

(8) Blocken, B.: Carmeliet, J.: "High-resolution wind-driven rain measurements on a low-rise building-experimental data for model development and model validation". J. Wind Eng. and Ind. Aerodinamics. vol. 93,12 (2005) págs. 905/928.

(9) Best, A.C.: "The size distribution of raindrops", Quarterly J. Roy. Met. Soc. 76, (1950) págs. 16-36.

(10) Laws, J. O. \& Parsons, D. A.: Relation of raindrop size to intensity. Amer. Geophys. Union Trans. 24 part II, (1943) pag. 453-460. 